\title{
AVALIAÇÃO DA ATENÇÃO PRIMÁRIA À SAÚDE EM UMA ESTRATÉGIA SAÚDE DA FAMÍLIA NO INTERIOR DO PARÁ: UTILIZAÇÃO DO PCATool-versão Brasil
}

\author{
Brenda Ramos de Souza, Jonatas Bezerra Tavares, Cláudia Cristina Pinto Girard, Ilma Pastana \\ Ferreira.
}

\section{RESUMO}

Motivação: A motivação para o estudo surgiu do questionamento de como era o desempenho da ESF como parte integrante da APS no município, gerando a primeira hipótese de bom desempenho da ESF e a segunda hipótese de uma ESF com escores não qualificadores.

Método: Trata-se de um estudo quantitativo, descritivo e transversal, realizado de janeiro a novembro de 2017 com 329 usuários de uma ESF no município de Tucuruí, no estado do Pará, onde foi aplicado um questionário adaptado chamado "Instrumento de Avaliação da Atenção Primária" (PCATool versão Brasil) para a coleta de dados. Os resultados foram analisados a partir do cálculo dos escores de cada atributo, sendo que as médias gerais acima de 6,6 foram consideradas de alto escore e as menores de baixo escore de acordo com a conversão para a escala de Likert.

Resultados: Os resultados apontaram que a ESF apresentou os seguintes escores qualificadores: 8,44 para o atributo acesso de primeiro contato-utilização e 7,66 para coordenação de sistemas de informação. Seguidos pelos baixos escore de: 5,84 para longitudinalidade, 5,31 para integralidade-serviços disponíveis, 3,93 para o acesso de primeiro contato-acessibilidade, 3,31 para coordenação-integração de cuidados, 2,68 para integralidade dos serviços prestados. Os atributos derivados de APS também foram considerados de baixo escore, cuja orientação familiar obteve escores de 4,66 e orientação comunitária de 4,11. Ao final, o escore geral foi de 5,69 e essencial de 5,31, considerados não qualificadores de APS.

Conclusões: 0 baixo escore geral e essencial revelaram a necessidade de mais planejamento dos serviços da ESF com base nos atributos qualificadores da APS, uma vez que são essenciais para um bom desempenho e a busca de maior satisfação dos usuários, fazendo-se necessário o esforço conjunto dos gestores, equipe e comunidade para implementação de novas práticas e mudanças voltadas a qualificação da APS pautadas em iniciativas avaliativas.

Palavras-chave: Atenção Primária à Saúde. Atenção Básica. Saúde da Família. Avaliação em Saúde. Gestão em saúde.
Revista da Rede APS 2019

Publicada em:

24/07/2019

DOI:10.14295/aps.v1i2.24

Brenda Ramos de Souza (Universidade do Estado do Pará, Brasil); Jonatas Bezerra Tavares

(Fundação Escola de Saúde Pública de Palmas, Tocantins, Brasil); Cláudia Cristina Pinto Girard (Universidade do Estado do Pará, Brasil); IIma Pastana Ferreira (Hospital Universitário João de Barros Barreto da Universidade Federal do Pará, Brasil)

Correspondência para: Brenda Ramos de Souza, brendaramosdesouza@g mail.com 
Vol. 1, n. 2, p. 112/120 | Maio/Agosto - 2019

ISSN 2596-3317 - DOI 10.14295/aps.v1i2.24

Souza, B. R.; Tavares, J. B.; Girard, C. C. P.; Ferreira, I.P

\section{INTRODUÇÃO}

Avaliar em saúde consiste na prática voltada a busca de qualidade que possibilita enxergar na realidade, indicadores reais da situação de saúde, permitindo também a intervenção local através de normas e critérios bem definidos. Para que a avaliação seja condizente com a realidade são necessários instrumentos que envolvam seus participantes no compromisso com as mudanças que precisem ser implementadas (BRUINKOOISTRA et al., 2012).

Pesquisas relacionadas a avaliação em saúde na Atenção Básica tiveram uma expansão significativa a partir de 2003, com 84 trabalhos voltados para essa temática (VERAS, VIANNA, 2009). Um dos primeiros projetos de avaliação da Atenção Básica no Brasil surge em 2005 com o nome "Avaliação para Melhoria da Qualidade da Estratégia Saúde da Família" (AMQ), essa metodologia estava voltada a autoavaliação e autogerenciamento (BRASIL, 2012).

No ano de 2007 é criado em São Paulo o Questionário Avaliação da Qualidade de Serviços de Atenção Básica (QualiAB) com intuito de avaliar todos os serviços de $A B$ independente do modelo de gestão da localidade o que possibilitava uma visão mais ampla do que a avaliação anterior (CASTANHEIRA et al.,2014).

Em 2010 o Ministério da Saúde (MS) parte do princípio de avaliar a APS através de suas características, adotando um instrumento de avaliação criado desde de 1998 nos Estado Unidos por Bárbara Starfield comumente conhecido como "PCATool - Primary Care Assessment Tool", sendo traduzido e validado para o Brasil como "Instrumento de Avaliação da Atenção Primária" (BRASIL, 2010).

O PCATool consiste em uma forma padronizada de avaliar a presença e extensão de atributos presentes na APS, caracterizados em essenciais (acesso de primeiro contato, longitudinalidade, integralidade e coordenação) e derivados (atenção à saúde, orientação comunitária e competência cultural), além de calcular através desses atributos, escore essencial e geral (BRASIL, 2010).

O resultado de estudos nessa linha avaliativa foi a criação do AMAQ (Autoavaliação para Melhoria) e o lançamento em 2011 do Programa de Avaliação para a Qualificação do SUS e o Programa Nacional de Melhoria do Acesso e da Qualidade da Atenção Básica (PMAQ). A partir desse momento, avaliar se torna um programa instituído e necessário para qualificação da $A B$, apresentando a autoavaliação como instrumento para a tomada de decisão (BRASIL, 2016).

Durante a adesão ao PMAQ, o estudo de Cavalcanti, Neto e Sousa (2015) buscou analisar os desafios apontados pelos gestores de 3.934 municípios do Brasil. Cada um deles listou cinco desafios de seu município, dos 35 problemas apontados, o eixo mais destacado pelos gestores da região Norte foi o desafio de avaliação, principalmente quando se trata em capacitar os recursos humanos na gestão local da $A B$ apenas com base em indicadores de saúde.

Diante dos esforços do programa PMAQ, e os diferentes tipos de avaliação, propõe-se avaliar o grau de orientação das ações da APS através do método de Starfield para verificar o perfil de atuação da APS na localidade estudada. Quanto maior o grau de orientação para APS, melhores serão os indicadores de saúde e maior será o grau de satisfação do serviço pelos usuários (SOUSA et al., 2016).

\section{MATERIAIS e MÉTOdos}

Trata-se de um estudo com abordagem quantitativa de caráter descritivo e delineamento prospectivo do tipo transversal, realizado de janeiro a novembro de 2017 e com método de análise estatístico-descritivo. Aprovado através do Comitê de Ética e Pesquisa da Universidade do Estado do Pará através do no 68196417.5.0000.5170.

Participaram 329 usuários cadastrados na ESF I do bairro Jardim Colorado, no município de Tucuruí (PA) através de uma amostragem aleatória simples 


\section{APS em Revista}

Vol. 1, n. 2, p. 112/120 | Maio/Agosto - 2019

ISSN 2596-3317 - DOI 10.14295/aps.v1i2.24

Souza, B. R.; Tavares, J. B.; Girard, C. C. P.; Ferreira, I.P

para uma média de 900 famílias cadastradas, considerando erro amostral de $5 \%$.

A coleta de dados foi realizada através de um questionário adaptado contendo a primeira parte com perfil sociodemográfico (idade, sexo, procedência, nível de escolaridade, ocupação e tempo que reside na localidade) e a segunda parte do questionário, sendo composta por 84 questões divididas em 9 componentes de acordo com os atributos essenciais e derivados do instrumento PCATool.

As respostas do instrumento foram divididas em "com certeza sim", "provavelmente, sim", "provavelmente, não", "com certeza, não" e "não sei ou não lembro" que correspondem respectivamente aos valores 4, 3, 2, 1 e 9, considerada com escala de Likert.

Inicialmente os dados foram codificados e tabulados através do programa Microsoft Office Excel 2013 na etapa pré-analítica, realizou-se a frequência absoluta e porcentagem das variáveis sociodemográficas e posteriormente foram calculadas as médias aritméticas por indivíduo e atributos, distribuindo os escores essencial e geral com a descrição estatística dos mesmos.

\section{RESUltadOS}

O perfil da amostra demonstrou a prevalência de 261 usuários pesquisados do sexo feminino (79,33\%), 149 na faixa etária entre 18 a 37 anos (45,29\%), 232 de cor parda (70,52\%), 182 provenientes do Pará $(55,32 \%), 156$ alfabetizados (47,42\%), 143 do lar (43,47\%), 288 morando com 1 a 6 pessoas $(87,54 \%)$ e 209 usuários morando em torno de 1 a 13 anos $(63,53 \%)$ no bairro Colorado.

Os indicadores da APS mostraram que os atributos avaliados como essenciais para ESF obtiveram as seguintes médias: acesso de primeiro contatoutilização pelos usuários com 8,44 (IC 8,38-8,51), acessibilidade com 3,93 (IC 3,41-3,98), longitudinalidade com 5,84 (IC 5,80-5,88), coordenação -integração de cuidados com 3,31 (IC 3,26-3,37), coordenação-sistemas de informação com 7,66 (IC 7,59-7,73), integralidade dos serviços disponíveis com 5,31 (IC 5,23-5,30) e 2,68 (IC 2,642,72 ) para integralidade dos serviços prestados (Tabela 2).

Tabela 2- Atributos essenciais e derivados do Modelo de APS de Bárbara Starfield aplicado na ESF. Tucuruí, PA, 2017.

\begin{tabular}{|c|c|c|c|c|c|}
\hline $\begin{array}{l}\text { Atributos da Atenção Primária a } \\
\text { Saúde }\end{array}$ & Escore Médio & Moda & Mediana & $\begin{array}{l}\text { Desvio } \\
\text { Padrão }\end{array}$ & IC (95\%) \\
\hline $\begin{array}{l}\text { A- Acesso de Primeiro Contato- } \\
\text { Utilização }\end{array}$ & $8,44^{*}$ & 4 & 4 & 1,01 & $(8,38-8,51)$ \\
\hline $\begin{array}{l}\text { B-Acesso de Primeiro Contato- } \\
\text { Acessibilidade }\end{array}$ & 3,93 & 1 & 1 & 1,30 & $(3,41-3,98)$ \\
\hline C-Longitudinalidade & 5,84 & 4 & 4 & 1,41 & $(5,80-5,88)$ \\
\hline $\begin{array}{l}\text { D-Coordenação-Integração de } \\
\text { Cuidados }\end{array}$ & 3,31 & 1 & 1 & 1,39 & $(3,26-3,37)$ \\
\hline $\begin{array}{l}\text { E-Coordenação-Sistemas de } \\
\text { Informação }\end{array}$ & $7,66^{*}$ & 4 & 4 & 1,12 & $(7,59-7,73)$ \\
\hline F-Integralidade-Serviços Disponíveis & 5,31 & 4 & 2 & 1,36 & $(5,23-5,30)$ \\
\hline G-Integralidade-Serviços Prestados & 2,68 & 1 & 1 & 1,80 & $(2,64-2,72)$ \\
\hline H- Orientação Familiar & 4,66 & 1 & 2 & 2,38 & $(3,89-5,43)$ \\
\hline I-Orientação Comunitária & 4,11 & 1 & 2 & 1,35 & $(4,06-4,17)$ \\
\hline Escore Essencial & & & 5,31 & & \\
\hline Escore Geral & & & 5,69 & & \\
\hline
\end{tabular}

*atributos com alto escore (>=6,6), ** IC: Intervalo de Confiança de 95\%. 
Vol. 1, n. 2, p. 112/120 | Maio/Agosto - 2019

ISSN 2596-3317 - DOI 10.14295/aps.v1i2.24

Souza, B. R.; Tavares, J. B.; Girard, C. C. P.; Ferreira, I.P

Observa-se que somente os atributos acesso de primeiro contato (utilização) e coordenação (sistemas de informação) obtiveram valores acima de 6,6. Essa relação pode ser mostrada na Figura 1 quanto mais distante do centro, mais qualificado o atributo da ESF.

Figura 1- Escores Médios dos atributos essenciais e derivados apresentados pela ESF. Tucuruí, PA, 2017.

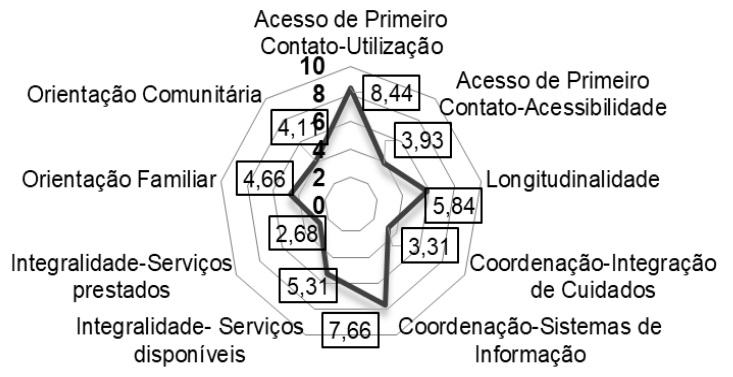

Os atributos derivados de APS também foram avaliados como baixo escore (não atingiram a média de 6,6). O atributo orientação familiar obteve média de 4,66 $(3,89-5,43)$ e orientação comunitária de 4,11 (IC 4,06-4,17), o que contribuiu para o aumento do escore essencial de 5,31 para um escore geral de 5,69, mas apesar do aumento, ainda permaneceu abaixo do considerado ideal para o modelo qualificador de APS.

\section{DISCUSSÃO}

O primeiro atributo proposto pelo instrumento de Starfield é o acesso, que está dividido em acesso de primeiro contato utilização e acessibilidade. Starfield (2002) trabalha o conceito do acesso de primeiro contato como a busca por atendimento pelos serviços como primeira escolha. De forma processual o usuário busca o serviço e de forma estrutural, o serviço responde ao usuário prestando o atendimento de modo conveniente a sua necessidade, criando circunstâncias que facilitem o retorno através da acessibilidade.

Oliveira (2013) considera acesso e a acessibilidade como conceitos discrepantes. O acesso consiste em permitir que as pessoas usem ou procurem a ESF ou unidade, enquanto que a acessibilidade considera os aspectos sócio-organizacionais do funcionamento dos serviços como fator interferente na relação com o usuário como: o tempo de espera, forma de comunicação, a dificuldade em conseguir atendimento, entre outros fatores de organização do serviço.

O resultado de alto escore para o acesso de primeiro contato-utilização $(8,44)$ e baixo escore para a acessibilidade $(3,93)$ foi congruente com o estudo de Oliva, Moura e Lima (2015) desenvolvido em Monte Carlos Minas Gerais, o que demonstra que os usuários estão procurando o serviço como primeira escolha de atendimento, mas a ESF não está fornecendo meios eficazes para a facilitação do acesso aos seus serviços, nem eliminando possíveis barreiras de acessibilidade.

Da mesma forma, a acessibilidade é considerada o principal fator para garantir uma satisfação mais positiva do serviço e por isso uma das dimensões mais difíceis de ser alcançada (PAIVA et al., 2015), justificando o resultado desse estudo.

$\mathrm{O}$ atributo longitudinalidade também avaliado com baixo escore, é conceituado por Fagá (2016) como a relação do profissional e paciente que se perpetua para garantir um vínculo entre ambos e por isso é considerado um dos atributos mais importantes. Além da confiança entre a população e o serviço, é necessário garantir que após o acesso de primeiro contato, este usuário continue procurando a ESF como primeira escolha de atendimento (BRASIL, 2010).

Segundo o estudo de Moraes, Campos e Brandão (2014), a rotatividade profissional é um fator que contribui negativamente para o vínculo. Em seu estudo, observou-se que a maioria dos usuários fazia referência aos médicos mais antigos e tinham maiores dificuldades em ter relacionamento mais duradouros com os médicos mais novos, como também foi constatado neste estudo.

Outro atributo considerado por Starfield (2002) é a coordenação de atenção, subdividido em coordenação integração de cuidados, coordenação sistemas de informação, o primeiro avaliado com baixo escore e o segundo com alto escore. Segundo a mesma autora, coordenação consiste 
Vol. 1, n. 2, p. 112/120 | Maio/Agosto - 2019

ISSN 2596-3317 - DOI 10.14295/aps.v1i2.24

Souza, B. R.; Tavares, J. B.; Girard, C. C. P.; Ferreira, I.P

nos meios para garantir que o usuário continue sendo atendido na rede de saúde, seja através do atendimento pelo mesmo profissional e/ou sistemas de informação com os dados do usuário.

A coordenação de cuidados considerada como uma das diretrizes para a $A B$, funciona como o processo de articulação entre os diferentes pontos da rede de atenção à saúde para garantir o fluxo dos usuários. Cabe à ela a responsabilidade de encaminhamento, planejamento, organização e o acompanhamento do usuário por meio de uma gestão compartilhada (BRASIL, 2017).

O resultado negativo da coordenação de atenção pode estar relacionado com o pouco contato do profissional com a rede de atendimento especializado e sobre o tempo elevado de espera para a marcação de consulta com especialista que acaba por dificultar o retorno para a integração do atendimento pelo profissional da AB (ALMEIDA, MARIN, CASOTTI, 2017).

Outro ponto discutido é a dificuldade da fixação do profissional nas USF's, uma vez que quando o usuário retorna da consulta com o especialista, o mesmo é atendido por outro profissional e que em alguns casos não tem perfil para trabalhar na APS. Desta forma, a falta de longitudinalidade incide diretamente na coordenação do cuidado, como também foi constatado no presente estudo (SANTOS, GIOVANELLA, 2016).

A dificuldade de referência e contrarreferência resulta na dificuldade para o retorno dos usuários às unidades de $A B$, principalmente porque as unidades de referência especializada não se comprometem em incentivar o retorno do usuário ao serviço de APS para garantir a continuidade do cuidado (MASIGLIA, 2012).

Como percebido, a coordenação de cuidados através do sistema de informações foi julgada positivamente, resultando a necessidade de articulação organizacional da rede de atenção. Como tentativa de melhorar esse problema a atual PNAB recomenda a articulação da rede mediante centrais de regulação em cada UBS com o estabelecimento de protocolos de encaminhamento ao serviço especializado e contato com as demais UBS's, sendo de total responsabilidade da gestão municipal, a ordenamento destes fluxos (BRASIL, 2017).

O quarto atributo consiste na integralidade, conceituado pela reunião das ações de saúde no campo da promoção, proteção, prevenção, diagnóstico, tratamento, reabilitação, redução de danos e manutenção de saúde (BRASIL, 2017). Na atual PNAB, são acrescentados à $A B$ as ações de cuidados paliativos e vigilância em saúde. Ambos os textos destacam o desenvolvimento dessas ações como prática de "cuidados integrados" (BRASIL, 2012).

Como verificado neste estudo os serviços mais comuns foram reconhecidos como presente na unidade, dentre eles os mais retratados foram: imunização, serviços odontológicos, pré-natal, coleta de exame citopatologico. Serviços menos desenvolvidos foram: colocação de tala em caso de fratura, remoção de verruga e aconselhamento contra o uso de drogas, serviços não muito comuns com a realidade da APS.

Por outro lado, o baixo escore da integralidade de serviços prestados está relacionada a pouca discussão de temas como: segurança do lar, uso de cinto de segurança, conflitos familiares, queimaduras, quedas, exposição a substâncias perigosas, saúde alimentar, entre outros temas sociais referidos pelos usuários no estudo.

A inserção de temas sociais como tratados anteriormente nas consultas dos profissionais da APS está distante da realidade brasileira, principalmente devido as dificuldades com a promoção de saúde. Neste âmbito, a APS deve articular diversos temas que saem do campo médico, para assim, promover mudanças de hábitos de vida em virtude da saúde. A discussão de temas sociais devem partir da necessidade da população e o envolvimento de toda a comunidade na discussão dos mesmos (SODRÉ, 2014).

$\mathrm{Na}$ atual PNAB, considera-se que o contexto das famílias são importantes no cuidado centrado a pessoa e traz consigo um conjunto de fatores 
Vol. 1, n. 2, p. 112/120 | Maio/Agosto - 2019

ISSN 2596-3317 - DOI 10.14295/aps.v1i2.24

Souza, B. R.; Tavares, J. B.; Girard, C. C. P.; Ferreira, I.P

intervenientes nos processos de desenvolvimento da vida, por tomar como base a família, a ESF tem como dentre outros princípios, a territorialização e adiscrição da população de forma a fortalecer a proximidade com a vida familiar e comunitária (BRASIL, 2017).

Escores considerados baixos no atributo orientação também foram encontrados em outros estudos como o de Alencar e colaboradores (2014) ao qual atribui o resultado ao desconhecimento dos usuários EM relação a gestão participativa, oferta de serviço de saúde e intersetorialidade. Nesse sentido, a gestão da ESF também deve apontar para questões de participação comunitária e controle social em suas ações de base, além da avaliação intersetorial do contexto familiar.

\section{CONCLUSÃO}

Avaliar a presença de atributos essenciais e derivados, permitiu verificar que a ESF do município de Tucuruí apresenta algumas falhas como atuante na APS, evidenciadas pelo baixo escore dos atributos essenciais e geral. Ao mesmo tempo a presença de alguns atributos qualificadores de acesso de primeiro contatoutilização e coordenação sistema de informações reflete a necessidade da constante avalição dos serviços e na possibilidade de implantação de estratégias de mudanças para o alcance dos demais atributos qualificadores da APS dentro das ESF's.

Os escores negativos presentes neste estudo, indicam a necessidade de maiores investimentos e aperfeiçoamento de muitas características consideradas essenciais para o padrão da APS, tal como está preconizada na própria Política de Atenção Básica brasileira, além do incentivo às novas formas de avaliação.

\section{REFERÊNCIAS BIBLIOGRÁFICAS}

(1) ALENCAR, Monyk Neves de et al. Avaliação do enfoque familiar e orientação para a comunidade na Estratégia Saúde da Família. Ciênc. saúde coletiva, Rio de Janeiro , v. 19, n. 2, p. 353-364, Feb. 2014 Disponível em: < http://www.scielo.br/scielo.php?pid=\$1413$81232014000200353 \&$ script=sci_abstract\&tIng=es >. Acesso em 27 fev. 2019.

(2) ALMEIDA, Patty Fidelis de; MARIN, Juliana; CASOTTI, Elisete. Estratégias para consolidação da coordenação do cuidado pela atenção básica. Trab. educ. saúde, Rio de Janeiro , v. 15 , n. 2, p. 373398, 2017 . Disponível em: < http://www.scielo.br/scielo.php?script=sci_arttext \&pid=S198177462017000200373\&lng=en\&nrm=iso\&tlng=pt >. Acesso em: 30 jan. 2019.

(3) BRASIL. Diretrizes para Organização das Redes de Atenção à Saúde do SUS. Brasília: Grupo Técnico da Comissão Intergestores Tripartite; 2010. Disponível em: <http://portalarquivos2.saude.gov.br/images/pdf/2 016/maio/18/2-B---Documento-de--Diretrizes-paraOrganiza----o-das-Redes-de-Aten----o----Sa--de-doSUS.pdf>. Acesso em: 15 mar. 2019.

(4) BRASIL. Ministério da Saúde. Autoavaliação para melhoria do Acesso de Qualidade. 3 ed. Brasília: Ministério da Saúde; 2016. Disponível em: < http://bvsms.saude.gov.br/bvs/publicacoes/autoav aliacao_melhoria_acesso_qualidade_amaq_2ed.pdf >. Acesso em: 28 mar.2019.

(5) BRASIL. Ministério da Saúde. Manual do instrumento de avaliação da atenção primaria à saúde. Brasília: Ministério da saúde; 2010. Disponível em:<http://189.28.128.100/dab/docs/publicacoes/ geral/manual_instrumento_avaliacao.pdf $>$. Acesso em: 7 jan. 2018.

(6) BRASIL. Ministério da Saúde. Portaria n².436, de 26 de novembro de 2017. Institui a Política Nacional de Atenção Básica. Disponível em: <http://www.foa.unesp.br/home/pos/ppgops/port aria-n-2436.pdf> Acesso em: 7 nov. 2017.

(7) BRASIL. Política Nacional de Atenção Básica. Brasília: Ministério da Saúde; 2012. Disponível em: 
Vol. 1, n. 2, p. 112/120 | Maio/Agosto - 2019

ISSN 2596-3317 - DOI 10.14295/aps.v1i2.24

Souza, B. R.; Tavares, J. B.; Girard, C. C. P.; Ferreira, I.P

<http://189.28.128.100/dab/docs/publicacoes/gera l/pnab.pdf >.Acesso em 7 nov.2017.

(8) BRASIL. Saúde mais perto de você: acesso e qualidade -Programa nacional de melhoria do acesso e da qualidade da atenção básica (PMAQ). Brasília, 2012. Disponível em: < http://189.28.128.100/dab/docs/publicacoes/geral/ manual_instrutivo_pmaq_site.pdf>. Acesso em 29 jan. 2019.

(9) BRUIN-KOOISTRA, Mieneke de.; AMELINKVERBURG, Marianne;BUITENDIJK, Simone E. Finding the right indicators for assessing quality midwifery care. International Journal for Quality in Health Care, v. 24, n. 3, p. 301-310, 2012. Disponível em: < https://academic.oup.com/intqhc/article/24/3/301 /1798786 >. Acesso em 15 Mar. 2019.

(10) CASTANHEIRA, Elen Rose Lodeiro et al . Avaliação de serviços de Atenção Básica em municípios de pequeno e médio porte no estado de São Paulo: resultados da primeira aplicação do instrumento QualiAB. Saúde debate, Rio de Janeiro , v. 38, n. 103, p. 679-691, 2014 . Disponível em: < http://www.scielo.br/scielo.php?script=sci_arttext \&pid=S0103-

$11042014000400679 \&$ Ing=en\&nrm=iso\&tlng=pt $>$. Acesso em: 20 fev. 2019.

(11) CAVALCANTI, Pauline Cristine da Silva; OLIVEIRA NETO, Aristides Vitorino de; SOUSA, Maria Fátima de. Quais são os desafios para a qualificação da Atenção Básica na visão dos gestores municipais?. Saúde debate, Rio de Janeiro , v. 39, n. 105, p. 323-336, June 2015 . Available from <http://www.scielo.br/scielo.php?script=sci_arttext \&pid=S0103-

$11042015000200323 \& \operatorname{lng}=e n \& n r m=i s o>$. Acesso em: 20 mar. 2019.

(12) FAGÁ, Maria de Almeida. Estudo dos atributos de Atenção Primária na rede de saúde de São Carlos - SP.2016. 121 f. Dissertação (Mestrado da Gestão da Clínica). Universidade Federal. São Paulo, São Carlos, 2016.

(13) MARSIGLIA, R.M.G. Universalização do Acesso ao Sistema Único de Saúde no Brasil: desafios para a Atenção Primária à Saúde. Cad. Ter. Ocup., São Carlos, v. 20, n. 3, p. 317-325, 2012. Disponível em: $<$ http://www.cadernosdeterapiaocupacional.ufscar. br/index.php/cadernos/article/view/676/390 >. Acesso em:

(14) MORAES, Verena Duarte; CAMPOS, Carlos Eduardo Aguilera; BRANDAO, Ana Laura. Estudo sobre dimensões da avaliação da Estratégia Saúde da Família pela perspectiva do usuário. Physis, Rio de Janeiro, v. 24, n. 1, p. 127-146, Mar. 2014. Available

from $<$ http://www.scielo.br/scielo.php?script=sci_arttext \&pid=S0103-

$73312014000100127 \&$ Ing=en\&nrm=iso $>$. access on 31 Mar. 2019.

(15) OLIVA, Ana Carolina Dias et al. Avaliação dos atributos do cuidado primário de saúde na perspectiva do usuário. Revista UNIABEU.v.8, n.18, p.196-208, 2015. Disponível em: < http://revista.uniabeu.edu.br/index.php/RU/article /view/1750/pdf_191 >. Acesso em: 31 de Mar. 2019.

(16) OLIVEIRA, Maria Amélia de Campos; PEREIRA, lara Cristina. Atributos essenciais da Atenção Primária e a Estratégia Saúde da Família. Rev. bras. enferm., Brasília, v. 66, n. spe, p. 158-164, 2013. Available from < http://www.scielo.br/pdf/reben/v66nspe/v66nspea 20.pdf >. Acesso em 29 jan. 2019.

(17) PAIVA, Marcele Bocater Paulo de et al. Uma contribuição para a avaliação da Atenção Primária à Saúde pela perspectiva do usuário. Physis, v. 25, n. 3, p. 925-950, 2015. Disponível em: $<$ http://www.scielo.br/scielo.php?script=sci_arttext \&pid=S0103-

$73312015000300925 \& \operatorname{lng}=e n \& n r m=i s o>$.Acesso em 20 de Março de 2019.

(18) SANTOS, Adriano Maia dos; GIOVANELLA, Ligia. Estratégia Saúde da Família na coordenação do cuidado em região de saúde na Bahia. Saúde debate, Rio de Janeiro, v. 40, n. 108, p. 48-63, Mar. 2016 Available from < http://www.scielo.br/scielo.php?script=sci_arttext \&pid=S0103- 
Vol. 1, n. 2, p. 112/120 | Maio/Agosto - 2019

ISSN 2596-3317 - DOI 10.14295/aps.v1i2.24

Souza, B. R.; Tavares, J. B.; Girard, C. C. P.; Ferreira, I.P

$11042016000100048 \&$ Ing=en $\&$ nrm=iso \&tlng=pt $\quad>$. access on 31 Mar. 2019.

(19) SODRÉ, F. O serviço social entre a prevenção e a promoção da saúde: tradução, vínculo $\mathrm{e}$ acolhimento. Serv. Soc. Soc., São Paulo, n. 117, p. 69-83, 2014.Disponível em: < http://www.scielo.br/pdf/sssoc/n117/05.pdf >. Acesso em: 31 de mar. 2019.

(20) SOUSA, Naira Pereira de et al . Internações sensíveis à atenção primária à saúde em hospital regional do Distrito Federal. Rev. Bras. Enferm., Brasília , v. 69, n. 1, p. 118-125, Feb. 2016. Available from <http://www.scielo.br/scielo.php?script=sci_arttext \&pid=S0034-

$71672016000100118 \&$ Ing=en\&nrm=iso $>$. Acesso em: 29 mar. 2019.

(21) STARFIELD, Bárbara. Atenção Primária: equilíbrio entre necessidades de saúde, serviços e tecnologia. Brasília: UNESCO/O ministério, 2002.Disponível em: < http://bvsms.saude.gov.br/bvs/publicacoes/atenca o_primaria_p1.pdf >. Acesso em 20 de jan.2019.

(22) VERAS, C.L.S.M.; VIANNA. R.P.T. Desempenho de Municípios paraibanos segundo avaliação de características da organização da atenção básica: Epidemiol. serv. Saúde, v.18, n.2, p. 133-140, 2009. Disponível em:

http://scielo.iec.gov.br/scielo.php?script=sci_arttex t\&pid=S1679-49742009000200004 >Acesso em: 14 fev. 2019. 


\section{ABSTRACT}

Objective: The study aimed to evaluate the performance of Primary Health Care (PHC) with the use of the PCATool-Brazil instrument applied in a Family Health Strategy (FHS) in the interior of Pará.

Background: The motivation for the study arose from the questioning of how the FHS performance was an integral part of the PHC in the municipality, generating the first hypothesis of good FHS performance and the second hypothesis of FHS with non-qualifying scores.

Methods: This is a quantitative, descriptive and cross-sectional study carried out from January to November $\mathbf{2 0 1 7}$ with $\mathbf{3 2 9}$ users of a FHS in the municipality of Tucuruí, in the state of Pará, where an adapted questionnaire called "Primary Care Assessment Instrument" (PCATool version Brazil) for data collection. The results were analyzed from the calculation of the scores of each attribute, and the general averages above 6.6 were considered high score and the lowest low score according to the Likert scale.

Results: The results showed that the FHS presented the following qualifying scores: $\mathbf{8 . 4 4}$ for the first contact-use access attribute and $\mathbf{7 . 6 6}$ for coordination of information systems. Followed by low scores: $\mathbf{5 . 8 4}$ for longitudinality, $\mathbf{5 . 3 1}$ for integrality-services available, $\mathbf{3 . 9 3}$ for first contact access, 3.31 for coordination-integration of care, 2.68 for integral services provided. The attributes derived from APS were also considered low score, whose family orientation obtained scores of 4.66 and a community orientation of 4.11. In the end, the overall score was 5.69 and essential, of 5.31, considered non-APS qualifiers.

Conclusions: The low overall and essential score revealed the need for further planning of FHS services based on the APS qualifying attributes, since they are essential for good performance and the search for greater user satisfaction, making it necessary the joint effort of the users, managers, team and community to implement new practices and changes aimed at qualifying the PHC based on evaluative initiatives.

Keywords: Primary Health Care; Primary Care; Family Health; Health Evaluation. Health Management. 